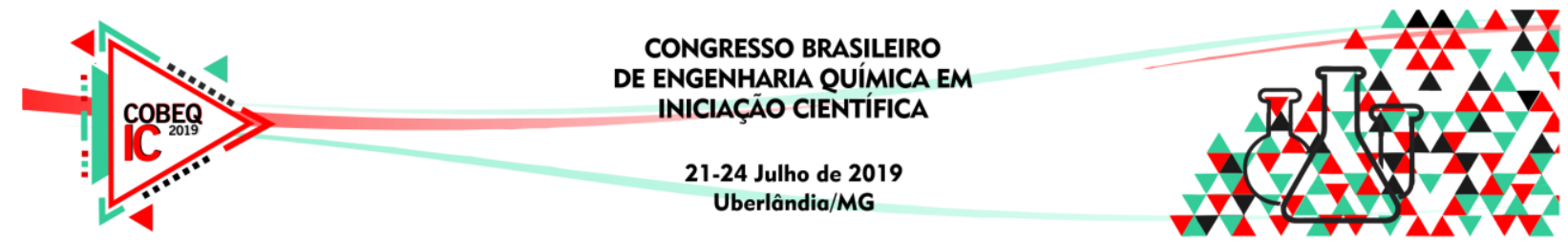

\title{
DEGRADAÇÃO POR PROCESSOS ELETROQUÍMICOS OXIDATIVOS AVANÇADOS DA CICLOFOSFAMIDA EM MEIO À URINA ARTIFICIAL
}

\author{
M. E. V. ALMEIDA ${ }^{1}$, B. S. SUGAHARA ${ }^{1}$, M. A. TONHELA ${ }^{1}$, A. C. G. MALPASS $^{1}$ e G. R. \\ P. MALPASS ${ }^{1}$
}

${ }^{1}$ Universidade Federal do Triângulo Mineiro, Instituto de Ciências Tecnológicas e Exatas E-mail para contato: veloso.eq@gmail.com

\begin{abstract}
RESUMO - A ciclofosfamida (CF) é um dos principais antineoplásicos utilizados no tratamento quimioterápico. Esse fármaco é excretado na urina, onde $25 \%$ da sua composição permanece inalterada, sendo inserido no meio ambiente através dos esgotos hospitalares. Por ser tóxico e pouco biodegradável pelas estações de tratamento, esse medicamento pode comprometer a qualidade da água e a saúde dos seres vivos. A fim de reduzir os impactos ambientais provocados, o presente trabalho avaliou a degradação da ciclofosfamida em meio de urina artificial, que apresenta altas concentrações de vários íons, por meio de processos eletroquímicos oxidativos avançados. Foram realizados testes preliminares em uma célula eletroquímica de bancada para estudar a influência das variáveis e seus respectivos níveis. Foram escolhidas como variáveis a corrente elétrica, vazão da bomba e tempo. As condições ótimas obtidas foram: corrente $(0,164$ A e 0,932 A), vazão $\left(7,022 \mathrm{~mL} \mathrm{~min}^{-1}\right)$ e intervalo de tempo (19 e $\left.37 \mathrm{~min}\right)$. Em condições otimizadas, a eficiência de diferentes técnicas combinadas (fotoeletroquímico, fotoquímico, sonoeletroquímico e sonoeletroquímico fotoassistido) foi avaliada. Os resultados obtidos comprovam a eficácia no tratamento do efluente através da combinação entre os processos fotoeletroquímico e sonoeletroquímico fotoassistido onde foi possível promover uma boa remoção tanto da espécie inicial quanto dos compostos orgânicos em solução.
\end{abstract}

\section{INTRODUÇÃO}

Monteiro (2001) caracteriza os fármacos antineoplásicos ou citostáticos como sendo quimioterápicos utilizados no tratamento de algumas patologias, dentre elas o câncer. Eles constituem uma categoria de fármacos cujo emprego está progressivamente aumentando, como causa do crescente número de casos diagnosticados de neoplasias.

Segundo Rang et al (2001), após atuarem no corpo humano, estes quimioterápicos serão eliminados nas excretas dos pacientes, sendo que no caso específico do agente antineoplásico ciclofosfamida, cerca de 5 a $25 \%$ do quimioterápico administrado é excretado na forma inalterada através da urina, participando da composição dos esgotos hospitalares e domésticos, visto que $90 \%$ desta eliminação ocorre no tempo de 24 horas. 


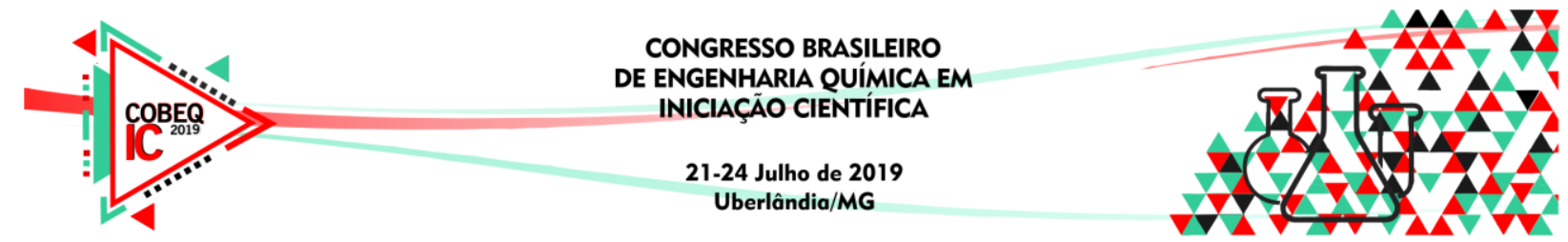

KÜMMERER et al (2000), constatou que muitos fármacos apresentam baixa biodegradabilidade e não são eliminados completamente pelas estações de tratamento de esgoto, o que representa um risco em potencial à saúde humana devido às suas propriedades mutagênicas, carcinogênicas e teratológicas.

De acordo com alguns autores, o tratamento de efluentes por oxidação eletroquímica avançada é um método alternativo de baixo custo para a eliminação de poluentes com boa eficiência (SANTOS et al., 2014; MARTÍNEZ-HUITLE et al., 2015) e que pode ser combinado com tratamentos convencionais já aplicados (GHAUCH, 2001).

Diante do contexto apresentado, o trabalho teve como motivação o estudo de técnicas avançadas capazes de promover a mineralização da ciclofosfamida no efluente assim como dos seus produtos de degradação com o propósito de reduzir os efeitos nocivos provocados ao meio ambiente e contribuir para a melhoria na qualidade de vida humana.

\section{METODOLOGIA}

Inicialmente, o sistema eletroquímico foi montado para realização dos testes iniciais. Fez-se o delineamento experimental e seguiu-se para a execução do planejamento por meio do planejamento fatorial $2^{\mathrm{k}}$ e do planejamento composto central (PCC) com objetivo de encontrar o ponto de resposta máxima na produção de espécies de cloro livre (ECL). As variáveis maximizadas foram aplicadas em diferentes técnicas eletroquímicas, avaliando demanda química de oxigênio (DQO), creatinina e ureia.

Os melhores parâmetros foram utilizados para os ensaios de degradação da ciclofosfamida e submetidos a análises de carbônico orgânico total (COT), cromatografia líquida de alta eficiência (CLAE) e consumo energético.

Os ensaios iniciais foram realizados em uma célula eletroquímica de bancada com fluxo em recirculação por meio de uma bomba peristáltica. $\mathrm{O}$ circuito foi ligado em uma fonte estabilizada. A temperatura foi mantida constante $\left(25^{\circ} \mathrm{C}\right)$ por um banho termostático acoplado a um reservatório da solução efluente. Nos ensaios fotoquímicos a irradiação UV foi fornecida por uma fonte luminosa de radiação UV, e nos ensaios sonoquímicos foi utilizado um banho ultrassom. Foi montado um sistema como mostrado na figura 1. 


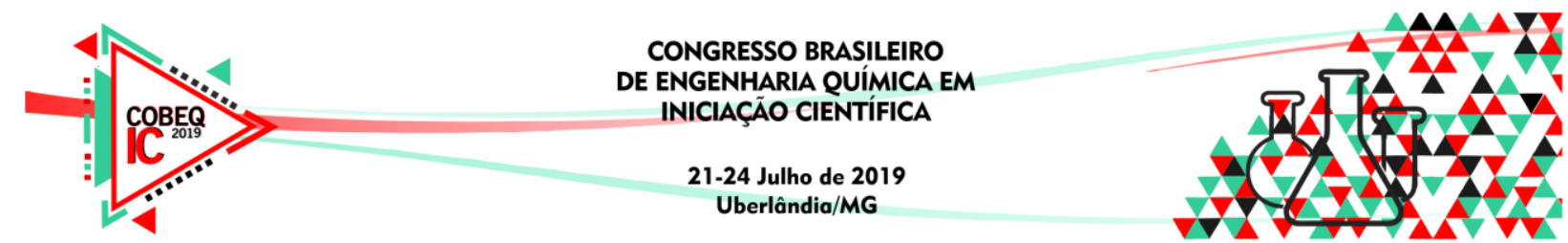

Figura 1- Esquema geral do sistema, sendo: (a) banho termostático; (b) reservatório da solução efluente;(c) bomba peristáltica (d) célula eletroquímica; (e) fonte estabilizada.

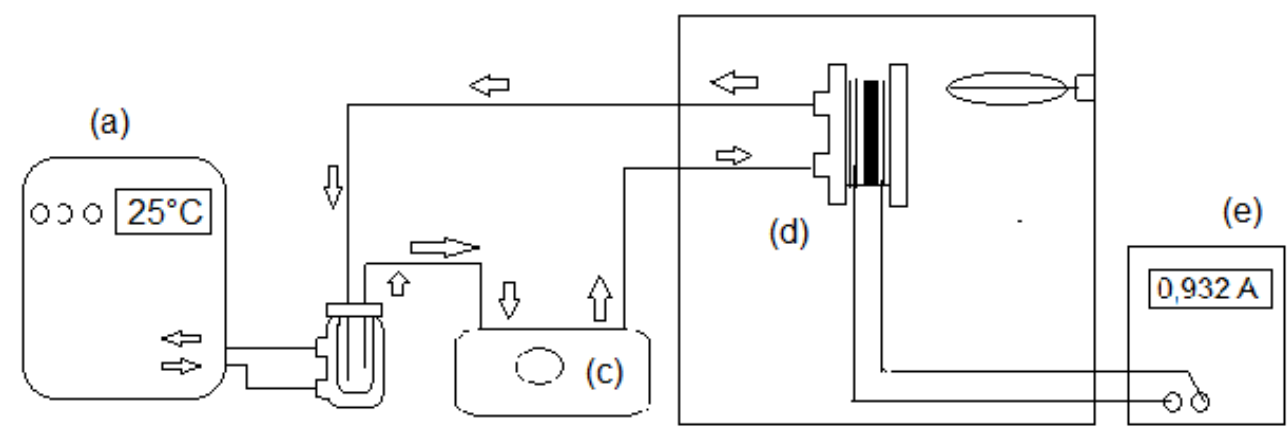

(b)

Fonte: Os autores, 2019.

\section{RESULTADOS}

\subsection{Planejamento Experimental}

Com os dados obtidos no planejamento fatorial $2^{3}$, identificou-se que o aumento na corrente e no tempo e a diminuição da vazão da bomba favorecem a produção de espécies de cloro livre, ou seja, com aumento do tempo e da corrente pode-se aumentar produção de cloro/ hipoclorito, isso ocorre por conta do maior tempo de residência dos íons cloretos no reator, gerando maiores concentrações de espécies de cloro livre (SINGLA, 2018). A vazão da bomba contribui negativamente tanto para produção de espécies de cloro livre quanto para eficiência energética, isto pode ser explicado, visto que o aumento da vazão desacelera a mineralização dos compostos orgânicos (FLORÊNCIO et al., 2014). Em termos de eficiência energética, obteve-se melhores resultados para baixos valores de vazão, corrente e tempo, justificando o estudo da questão energética do processo.

Após análise da significância das três variáveis de entrada e seus respectivos níveis, realizou-se o planejamento composto central objetivando verificar a curvatura do plano. Nessa etapa a vazão da bomba foi fixada em 7,022 mL. $\mathrm{min}^{-1}$ sendo esse o menor valor atingido pela bomba peristáltica. Visto que, menores vazões ocasionam os melhores efeitos no processo tanto em termos de espécies de cloro livre quanto em termos da eficiência energética.

A análise da superfície de resposta e de contorno são mostrados na Figura 2, os pontos brancos são os pontos do planejamento do composto central realizados. Pode-se observar que, a região de ótimo (vermelho escuro) são evidentes, indicando que os níveis na faixa estudada foram significativos para o resultado do experimento. 


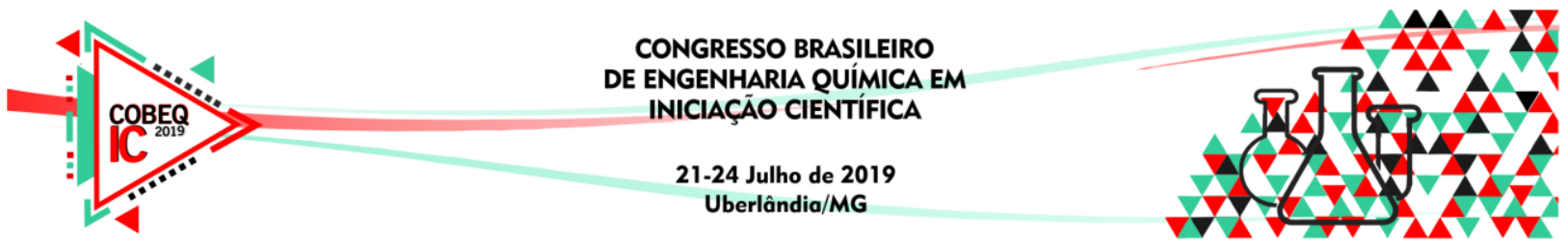

Figura 2- Gráficos de superfícies respostas para as variáveis obtidas a partir do planejamento composto central: (a) em função ao máximo de ECL e (b) em função à maior eficiência energética $(\mathrm{EE} \%)$.

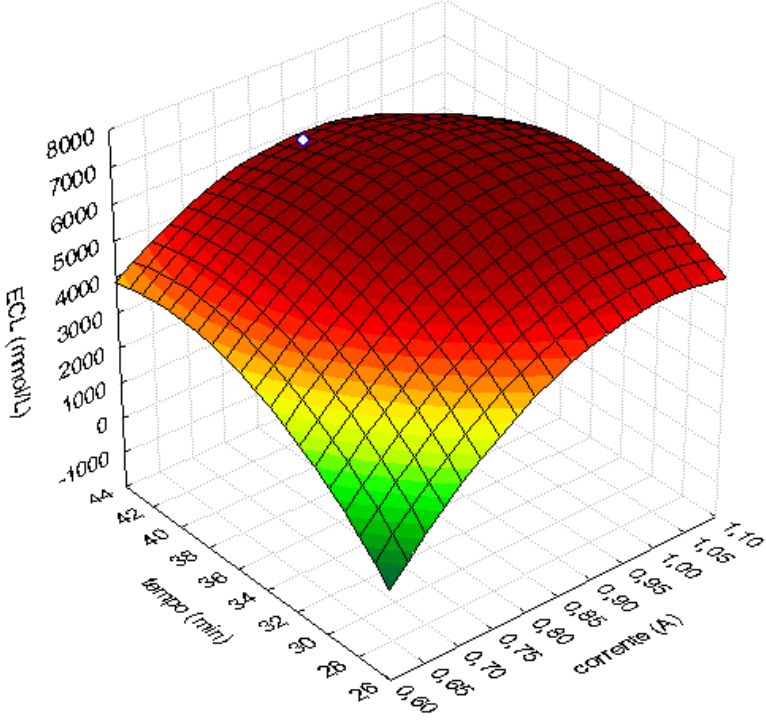

(a)

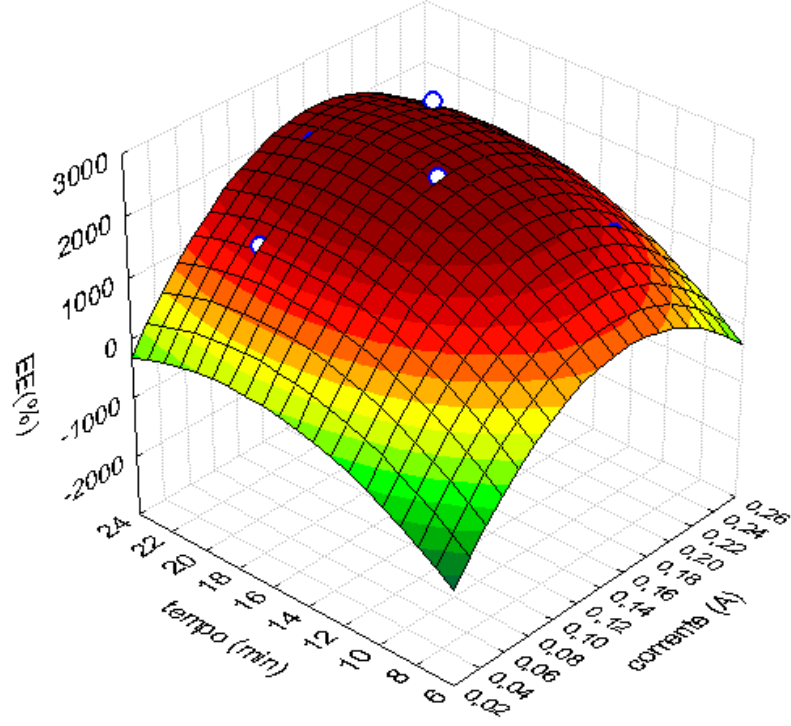

(b)

Fonte: Dos autores, 2019.

Uma vez que o modelo proposto foi satisfatório, determinou-se os pontos ótimos, sendo 0,932 A e 37 min em termos de produção máxima de ECL $\left(425,28 \mathrm{mg} \mathrm{L}^{-1}\right)$, e 0,164 A e 19 min em termos de eficiência energética $\left(212 \mathrm{mg} \mathrm{L}^{-1}\right)$. Essas condições foram aplicadas nos ensaios de degradação.

\subsection{Análise da Urina}

A creatinina, ureia, DQO e ECL foram analisadas dentro das condições otimizadas (0,932 A, $37 \mathrm{~min})$ e (0,164 A, 19 min) obtidos pelo planejamento experimental, empregando técnicas isoladas e combinadas (eletroquímica, fotoquímica, fotoeletroquímica, sonoeletroquímica e sonoeletroquímica fotoassistida). Nessas condições as técnicas fotoeletroquímicas e sonoeltroquímicas apresentaram maiores gerações de espécies oxidantes, uma vez que a irradiação do ultrassom e luz UV apresentam efeitos positivos nos resultados, levando a menores cargas elétricas para a mineralização completa dos resíduos (SOUZA et al., 2014). Favorecendo melhores remoções dos compostos orgânicos contidos na urina, obtendo remoções de creatinina e ureia 12,34 e 6,20\% para a técnica fotoeletroquímica com maior corrente $(0,932 \mathrm{~A}, 37 \mathrm{~min})$, enquanto que com a corrente menor $(0,164 \mathrm{~A}, 19 \mathrm{~min}) \mathrm{a}$ concentração de materiais orgânicos diminui $10,26 \%$ para creatinina e 4,46\% de remoção de ureia. Os resultados de DQO foram condizentes com os resultados de remoção das matérias orgânicas presentes na urina simulada (creatinina e ureia) no qual essas duas técnicas foram mais eficientes. 


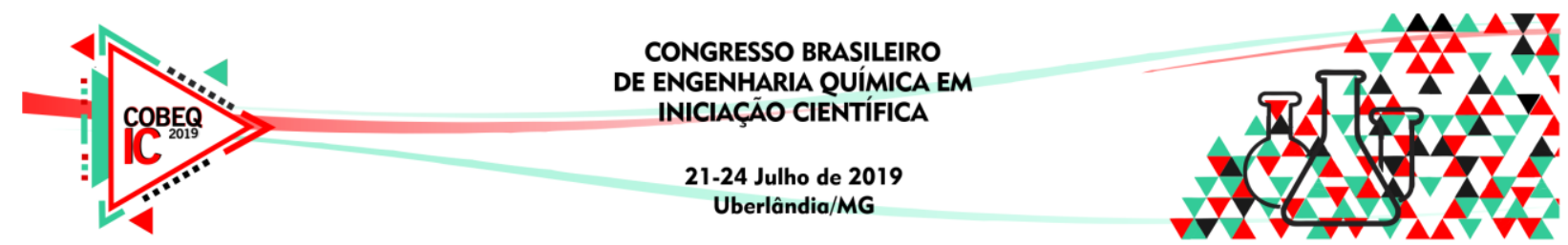

\subsection{Ensaio de Degradação}

Foi realizado um tratamento com essas duas melhores técnicas com uma faixa de tempo de reação de 180 minutos, para corrente em $0,164 \mathrm{~A}$ e $0,932 \mathrm{~A}$ afim de evidenciar a degradação da matéria orgânica contida na urina (creatinina e ureia) e a ciclofosfamida em relação a uma variação crescente do tempo.

A diminuição do teor de COT (Carbono Orgânico Total) indica a mineralização da urina sintética juntamente com o efluente hospitalar. O processo fotoeletroquímico mostrou-se o mais eficiente com redução de $5800 \mathrm{mg} . \mathrm{L}^{-1}$ para $3532 \mathrm{mg} . \mathrm{L}^{-1}$ do efluente bruto (aproximadamente 39,1\%) utilizando corrente de 0,932A e intensidade de luz UV de 400W. Para corrente em 0,164 A reduziu aproximadamente $22 \%$ para o processo fotoeletroquímico com intensidade de luz UV de $250 \mathrm{~W}$ ao longo de 180 minutos. O resultado é explicado pelo aumento na produção de $\mathrm{O}_{2}$ na presença de radiação $\mathrm{UV}$, causando uma maior oxidação da matéria orgânica (CASTANHO et al., 2006).

Nas degradações pelos processos fotoeletroquímico e sonoeletroquímico fotoassistido ambos a 400W ocorreram maiores remoções de ciclofosfamida de aproximadamente 77,46\% e 74, 87\% até 120 minutos. Em todas as condições experimentais, o processo de diminuição da concentração do fármaco investigado seguiu uma cinética de reação de pseudo-primeira ordem, que permitiu determinados valores de k' e observar que este valor aumenta em correntes e intensidade da irradiação da luz UV mais altas, esse aumento pode ser atribuído à aceleração da formação de radicais livres durante a reação aumentando a taxa de remoção da ciclofosfamida (SIEDLECKA et al., 2018).

Visando um menor consumo energético demandado pela degradação é recomendável trabalhar com correntes mais baixas ( $22 \%$ de remoção de COT), obtendo $3006 \mathrm{kWhm}^{-3}$ e custo de $\sim \mathrm{R} \$ 2,81$. A dificuldade de se atingir uma porcentagem maior de remoção de COT se dá pelo fato que exista competições de espécies reduzindo assim a eficiência da formação de cloro.

\section{CONCLUSÃO}

Foi possível verificar no desenvolvimento do presente estudo, empregando os parâmetros ótimos de corrente, vazão e tempo determinados no planejamento composto central, que dentre as diferentes técnicas estudadas, a fotoeletroquímica e a sonoeletroquimica fotoassistida apresentaram os melhores resultados tanto para a remoção de ureia e creatinina quanto para a ciclofosfamida. Além disso, também permitiu uma análise da viabilidade econômica do processo, através da comparação em termos de eficiência energética.

\section{REFERÊNCIAS}

CASTANHO, M.; MALPASS, G. R. P.; MOTHEO, A. DE J. Avaliação dos tratamentos eletroquímico e fotoeletroquímico na degradação de corantes têxteis. Química Nova, v. 29, n.5, p.983-989, 2006. 


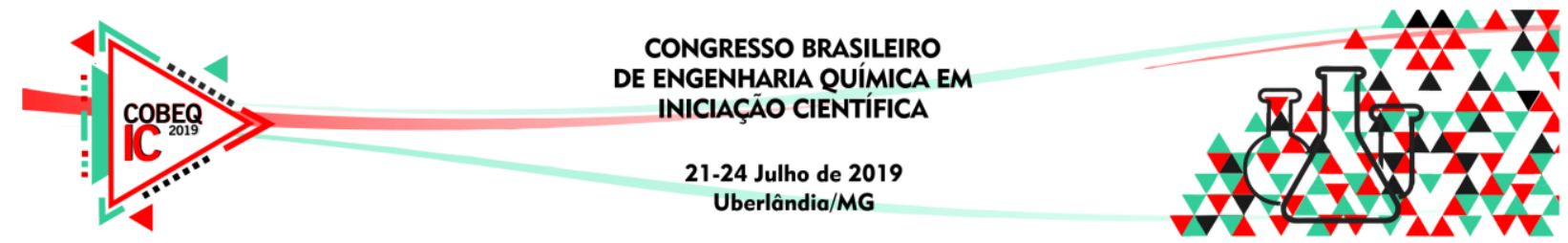

FLORÊNCIO, T, M.; DE ARAUJO, K, S.; ANTONELLI, R.; FORNAZARI, A. L. T, DA CUNHA, P. C. R.; DA SILVA, B. L.H.; MOTHEO, A, J, GRANATO, A, C.; MALPASS. Photo-assisted electrochemical degradation of simulated textile effluent coupled with simultaneous chlorine photolysis. Environmental Science and Pollution Research International, v. 23, p. 19292-19301, 2016.

GHAUCH, A.; GALLET, C.; CHAREF, A.; RIMA, J, MARTIN-BOUYER, M. Reductive degradation of carbaryl in water by Zero-valent iron A. Chemosphere v.42, p.419-424, 2001.

KÜMMERER, K.; AL-AHMAD, A.; BERTRAM, B. e WIE_LER, M. Biodegradability of antineoplastic compounds in screening tests: influence of glucosidation and of stereochemistry. Chemosphere; 40: 767-773, 2000.

MARTÍNEZ-HUITLE, C. A.; BRILLAS, E. Decontamination of wastewaters containing synthetic organic dyes by electrochemical methods. An updated review. Applied Catalysis B: environmental v.166, p.603-643, 2015.

MONTEIRO, A.B.C. Biossegurança no preparo, administração e descarte de agentes antineoplásicos injetáveis. Dissertação (Mestrado em Enfermagem) - Universidade de São Paulo/Ribeirão Preto, 2001.

RANG, H.P., DALE, M.M. e RITTER, J.M. Farmacologia. Guanabara Koogan, $4^{\circ}$ Ed. Rio de Janeiro, 2001.

SANTOS, D.; LOPES, A.; PACHECO, M.J.; GOMES, A.; CIRÍACO, L. The Oxygen Evolution Reaction at Sn-Sb Oxide Anodes: Influence of the Oxide Preparation Mode. Journal of The Electrochemical Society, v.161, n.9, p.564-572, 2014.

SIEDLECKA, E. M.; OCHOA- CHAVEZ, A.S.; PIECZYNSKA, A.; FISKA, B. A.; ESPINOZA- MONTERO, P. J. Electrochemical degradation of 5-FU using a flow reactor with BDD electrode: Comparison of two electrochemical systems. Chemosphere, v. 201, p. 816-825, 2018.

SINGLA, J.; VERMA, A.; SANGAL, V. K. Parametric optimization for the treatment of human urine metabolite, creatinine using electro-oxidation. Journal of Electroanalytical Chemistry, v. 809, p. 136-146, 2018.

SOUZA, F.L., SÁEZ, C., CANIZARES, P., MOTHEO, A.J., RODRIGO, M.A. Coupling photo and sono technologies to improve efficiencies in conductive diamond electrochemical oxidation. Applied Catalysis B: Environmental, v.144, p. 121-128, 2014. 